\title{
Hot star wind models with new solar abundances
}

\author{
J. Krtička ${ }^{1}$ and J. Kubát ${ }^{2}$ \\ 1 Ústav Teoretické Fyziky a Astrofyziky PřF MU, 61137 Brno, Czech Republic \\ e-mail: krticka@physics.muni.cz \\ 2 Astronomický Ústav, Akademie Věd České Republiky, 25165 Ondřejov, Czech Republic
}

Received 20 November 2006 / Accepted 11 January 2007

\begin{abstract}
We compare the hot-star wind models calculated by assuming older solar-abundance determination with models calculated using the recently published values derived from 3D hydrodynamical model atmospheres. We show that the use of new abundances with lower metallicity improves the agreement between wind observation and theory in several aspects. (1) The predicted wind massloss rates are lower by a factor of 0.76 . This leads to better agreement with mass-loss rates derived from observational analysis that takes the clumping into account. (2) As a result of the lowering of mass-loss rates, there is better agreement between the predicted modified wind momentum-luminosity relationship and that derived from observational analysis that takes the clumping into account. (3) Both the lower mass fraction of heavier elements and lower mass-loss rates lead to a decrease in opacity in the X-ray region. This influences the prediction of the X-ray line profile shapes. (4) There is better agreement between predicted P V ionization fractions and those derived from observations.
\end{abstract}

Key words. stars: winds, outflows - stars: mass-loss - stars: early-type - hydrodynamics

\section{Introduction}

Massive hot stars lose a substantial part of their mass via their stellar winds. Wind parameters, especially the wind mass-loss rate (the amount of mass lost by the star per time unit), are the functions of basic stellar parameters, for example the stellar luminosity (see, e.g., Kudritzki \& Puls 2000; Krtička \& Kubát 2006, for a review). The wind mass-loss rate is the most important wind parameter, because the presence of strong hot-star winds influences not only the emergent spectra, but also, for example, stellar evolution (e.g., Chiosi \& Maeder 1986).

Since hot-star winds are mainly accelerated by the absorption (or scattering) of radiation in lines of heavier elements (like carbon, nitrogen, or iron), the radiative force and wind massloss rate also depend significantly on metallicity. With increasing metallicity, the mass-loss rate and the wind terminal velocity increase (Kudritzki \& Puls 2000; Vink et al. 2001; Krtička 2006). Frequently, the solar abundance values of Anders \& Grevesse (1989) are used for the wind-model predictions. However, the solar metallicity was recently reduced following the calculations by Asplund et al. (2005) based on improved (self-consistent) treatment of the outer solar convective region (using 3D hydrodynamical model atmospheres, Nordlund \& Dravins 1990; Steffen et al. 1995; Asplund 2004). The difference between older abundance determinations and recent, more realistic ones is very striking, as the estimate of the mass fraction of heavier elements decreased from 0.0194 to 0.0122 . Consequently, it would be interesting to test the influence of the new solar abundance determination on the wind models. To do so, we compare wind models calculated with both old and new abundance determinations.

\section{Basic assumptions}

\subsection{Wind models}

We used our own NLTE wind models to predict the hotstar wind structure (Krtička \& Kubát 2004, recent improvements in our calculations, especially the inclusion of the Auger ionization, will be described elsewhere). Our models enabled us to self-consistently solve the hydrodynamical equations in the spherically-symmetric, stationary, radiatively-driven stellar wind. The radiative force (in the Sobolev approximation, Castor 1974) and the radiative heating/cooling term were calculated using the solution of NLTE equations, for which we mainly used the atomic data available from the Opacity and Iron Projects (Seaton et al. 1992; Hummer et al. 1993). This enabled us to change the abundance of each element separately according to its new value, and not only via the common parameter called the metallicity. Using our method we were able to predict wind density and velocity structure, namely the wind mass-loss rate $\dot{M}$ and the wind terminal velocity $v_{\infty}$.

\subsection{Model stars}

For our analysis we selected O stars with $T_{\text {eff }} \lesssim 40000 \mathrm{~K}$ (see Table 1), for which the stellar parameters were derived using the models with line blanketing. Effective temperatures and radii were taken from Repolust et al. (2004, hereafter R04), Markova et al. (2004, hereafter M04), and Martins et al. (2005, hereafter M05). Stellar masses were obtained using evolutionary tracks either by us (using Schaller et al. 1992 tracks) or by M05. 
Table 1. Stellar parameters of the O stars.

\begin{tabular}{rrccccc}
\hline \hline Star & $\begin{array}{c}\text { HD } \\
\text { number }\end{array}$ & $\begin{array}{c}\text { Sp. } \\
\text { type }\end{array}$ & $\begin{array}{c}R_{*} \\
{\left[R_{\odot}\right]}\end{array}$ & $\begin{array}{c}M \\
{\left[M_{\odot}\right]}\end{array}$ & $\begin{array}{c}T_{\text {eff }} \\
{[\mathrm{K}]}\end{array}$ & Source \\
\hline$\xi$ Per & 24912 & O7.5IIIe & 14.0 & 36 & 35000 & R04 \\
$\iota$ Ori & 37043 & O9III & 21.6 & 41 & 31400 & M04 \\
15 Mon & 47839 & O7Ve & 9.9 & 32 & 37500 & M04 \\
& 54662 & O7III & 11.9 & 38 & 38600 & M04 \\
& 93204 & O5V & 11.9 & 41 & 40000 & M05 \\
$\zeta$ Oph & 149757 & O9V & 8.9 & 21 & 32000 & R04 \\
68 Cyg & 203064 & O8e & 15.7 & 38 & 34500 & R04 \\
19 Cep & 209975 & O9Ib & 22.9 & 47 & 32000 & R04 \\
\hline
\end{tabular}

Data sources: M04 - Markova et al. (2004), R04 - Repolust et al. (2004), M05 - Martins et al. (2005).

Table 2. Comparison of wind parameters calculated assuming Anders \& Grevesse (1989) and Asplund et al. (2005) solar abundances.

\begin{tabular}{rcccc}
\hline \hline $\begin{array}{c}\text { HD } \\
\text { number }\end{array}$ & $\begin{array}{c}\text { Anders \& Grevesse (1989) } \\
\dot{M} \\
{\left[M_{\odot} \mathrm{yr}^{-1}\right]}\end{array}$ & $\begin{array}{c}v_{\infty} \\
{\left[\mathrm{km} \mathrm{s}^{-1}\right]}\end{array}$ & $\begin{array}{c}\text { Asplund et al. (2005) } \\
{\left[\begin{array}{c}\dot{M} \\
{\left[M_{\odot} \mathrm{yr}^{-1}\right]}\end{array}\right.}\end{array}$ & $\begin{array}{c}v_{\infty} \\
{\left[\mathrm{km} \mathrm{s}^{-1}\right]}\end{array}$ \\
\hline 24912 & $5.7 \times 10^{-7}$ & 2440 & $4.4 \times 10^{-7}$ & 2270 \\
37043 & $7.3 \times 10^{-7}$ & 2440 & $6.2 \times 10^{-7}$ & 2340 \\
47839 & $3.7 \times 10^{-7}$ & 2450 & $2.2 \times 10^{-7}$ & 3080 \\
54662 & $1.1 \times 10^{-6}$ & 2350 & $7.9 \times 10^{-7}$ & 2190 \\
93204 & $1.8 \times 10^{-6}$ & 2490 & $1.3 \times 10^{-6}$ & 2290 \\
149757 & $5.9 \times 10^{-8}$ & 2310 & $4.7 \times 10^{-8}$ & 2040 \\
203064 & $7.4 \times 10^{-7}$ & 2270 & $5.7 \times 10^{-7}$ & 2080 \\
209975 & $1.0 \times 10^{-6}$ & 2510 & $8.4 \times 10^{-7}$ & 2430 \\
\hline
\end{tabular}

\section{Wind models with Asplund et al. solar abundance determination}

With decreasing metallicity the radiative force decreases and, consequently, the wind mass-loss rate also decreases. Moreover, the decrease in metallicity leads to a slight lowering of the wind terminal velocity (Kudritzki \& Puls 2000; Vink et al. 2001; Krtička 2006). Both these effects are apparent in Table 2, where we compare wind parameters calculated using different determinations of solar abundances (see also Fig. 1). On average, the mass-loss rate changes by a factor 0.76 . This roughly corresponds (the abundance changes were not uniform for all elements) to the dependence $\dot{M} \sim Z^{0.60}$, where $Z$ is the mass fraction of heavier elements. This dependence is slightly lower than the one reported by Vink et al. (2001) and Krtička (2006). Note, however, that these results are not directly comparable, since the relative change in the abundance of different elements is not uniform in this study, whereas results by both Vink et al. (2001) and Krtička (2006) were obtained for a uniform relative change in the abundance. On average, the wind terminal velocity also decreases with decreasing metallicity (see Fig. 1). However, note that the change in the terminal velocity due to the decrease in the metallicity is mostly lower than the error in the terminal velocity determination.

\section{Implications of new abundance determinations}

The next logical step would be to compare our predicted wind parameters (especially the mass-loss rates) with the quantities derived from observation. However, such a comparison is complicated by the effect of clumping.

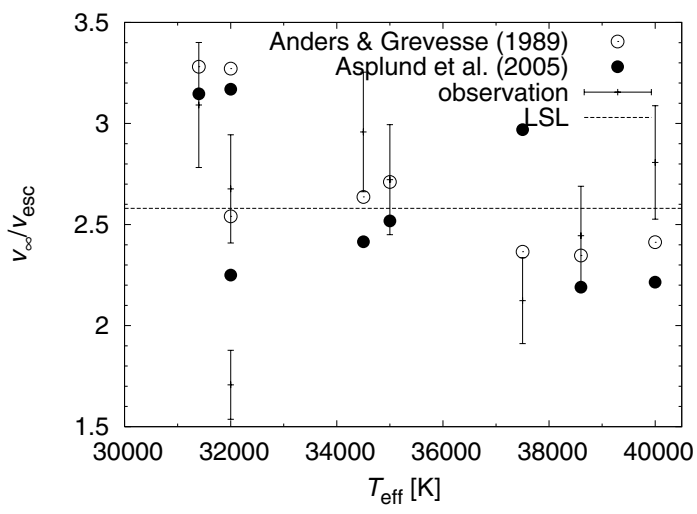

Fig. 1. Comparison of the ratio of the terminal velocity to the escape velocity calculated using terminal velocities either from theoretical calculations (with different sets of abundance determinations) or derived from observations (values adopted from Puls et al. 1996, M04, M05). LSL denotes the average ratio derived by Lamers et al. (1995).

\subsection{Wind clumping}

There are two ways to derive wind mass-loss rates. Since many spectral features are sensitive to wind density (or mass-loss rate), it is possible to derive their values from observations using wind models where the mass-loss rate is a free parameter. These values are sometimes called "observed", but we prefer to call them "derived from observations". On the other hand, the mass-loss rate can be calculated ab initio from dedicated wind models where stellar parameters (the effective temperature, mass, radius, and metallicity) serve as input parameters. This value of the mass-loss rate is usually called "predicted". It is clear that both ways of determining the wind mass-loss rate should be in close agreement.

However, recent spectroscopic studies (Bouret et al. 2003; Martins et al. 2005; Puls et al. 2006) indicate that there may be significant disagreement between the wind mass-loss rates derived from observations and predicted ones. The problem is that the emergent spectrum is, in many cases, not directly sensitive to the wind mass-loss rate $\dot{M}$, but to the wind density squared. This means that, if it happens that the wind is structured on small scales ("clumped"), then the lines originating in the clumped wind with a relatively low mean wind density may mimic those with a much higher mass-loss rate. If the winds are really significantly clumped in all regions from which we observe the emergent radiation, as seems to follow from the comparison of synthetic wind spectra with the observed ones (e.g., Bouret et al. 2003; Martins et al. 2005), then the real wind mass-loss rates are significantly lower than the predicted ones.

From the studies of wind spectra that include clumping (M05), it follows that wind mass-loss rates are roughly lower by a factor $\sim 0.3$ than those derived from the standard wind models of Vink et al. (2001). Consequently, the reduction of wind massloss rate by a factor 0.76 due to the reduced metallicity represents an improvement in the agreement between wind mass-loss rates derived from observation (that take the clumping into account) and predicted ones.

\subsection{Modified wind momentum-luminosity relationship}

It follows from both wind theory and observations that the value of the modified wind momentum $\dot{M} v_{\infty}\left(R / R_{\odot}\right)^{1 / 2}$ depends mainly on the stellar luminosity (Kudritzki \& Puls 2000, and references therein). Especially the mass-dependence nearly cancels 


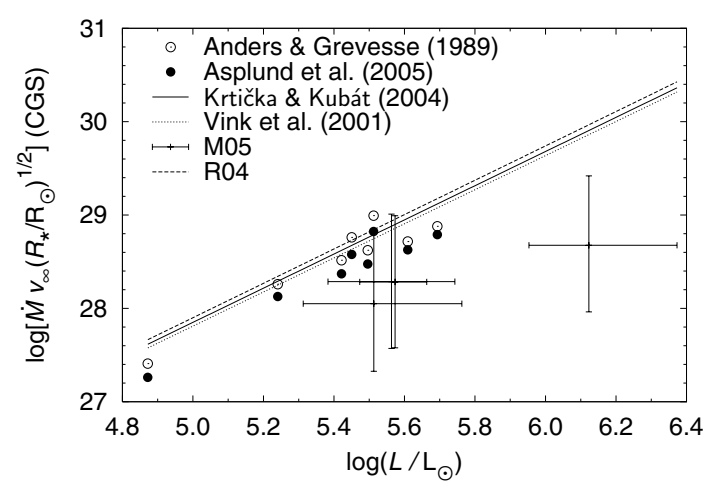

Fig. 2. Comparison of modified wind-momentum calculated for individual stars in this paper assuming solar abundances by Anders \& Grevesse (1989) with more recent lower ones by Asplund et al. (2005). We also plot the linear fits of the theoretical predictions, derived with older abundances (Krtička \& Kubát 2004; Vink et al. 2001), and values derived from observations both by M05 (individual stars for which the clumping was taken into an account) and by R04 (linear fit for giant and dwarfs, for which the clumping was not taken into an account).

out. Consequently, the wind momentum-luminosity relationship is suitable for comparing observations and theory. Here we compare this relationship derived using theoretical models with both older and new abundance determinations with values from M05, who derived wind mass-loss rates from the observed data and who account for clumping, and R04, who neglected the possibility of wind clumping (see Fig. 2).

The predicted wind momentum is lower for models calculated using improved solar metallicity determination, mainly due to the decrease in wind mass-loss rate with metallicity and, to a lesser extent, due to the lower terminal velocities that are derived. The theoretical modified wind momentum-luminosity relationship calculated using improved abundances has the form

$\log \left[\dot{M} v_{\infty}\left(R / R_{\odot}\right)^{1 / 2}\right]=1.91 \log \left(L / L_{\odot}\right)+18.1 \quad(\mathrm{CGS})$.

Our results to some extent improve the agreement between the theory and observational analysis that takes the clumping into account (M05 and also Fig. 2). However, they are not able to remove the difference between the theory and observations completely. Note that there is good agreement between theoretical predictions derived using Anders \& Grevesse (1989) abundances (Vink et al. 2001; Krtička \& Kubát 2004, and the empty circles in the Fig. 2) and observations by R04. Consequently, if the influence of clumping on the observed wind spectra is negligible, then the wind models with improved abundance determination predict lower wind momentum than those derived from observations.

\subsection{X-ray continuum optical depth}

Another painful problem of the hot-star wind theory is connected with the shape of X-ray line profiles. While the wind theory based on currently available theoretical mass-loss rates predicts asymmetric X-ray line profiles due to the continuum absorption within the wind (see, e.g., Owocki \& Cohen 2006; Oskinova et al. 2006), the observations mostly show symmetric X-ray line profiles. This indicates that the wind's optical depth in continuum for X-ray frequencies is small in the region where X-rays form.

To infer the impact of new solar abundances on the Xray line profiles, we compared the mass-absorption coefficients
Table 3. Comparison of continuum opacity (opacity per unit of mass in units of $\mathrm{cm}^{2} \mathrm{~g}^{-1}$ averaged for the radius $1.5 R_{\odot}<r<5 R_{\odot}$ ) in the $\mathrm{X}$-ray region for several different wavelengths calculated for the two abundance determinations (for HD 24912).

\begin{tabular}{lccccc}
\hline \hline wavelength [̊̊] & 8.1 & 12.0 & 15.8 & 20.0 & 24.0 \\
Anders \& Grevesse (1989) & 31 & 83 & 141 & 228 & 154 \\
Asplund et al. (2005) & 20 & 52 & 91 & 150 & 125 \\
\hline
\end{tabular}

calculated with solar abundances from both Anders \& Grevesse (1989) and Asplund et al. (2005). Apparently, since heavier elements dominate the continuum opacity in the X-ray region (especially due to Auger transitions), the mass absorption coefficient decreases with decreasing metallicity (see Table 3). The decrease in the optical depth is not equal for all wavelengths because different elements dominate the opacity for different wavelengths and the relative change of the metallicity is not the same for all elements. The influence of new solar abundance determination on the total continuum opacity in the X-ray region is amplified by the fact that the wind density also decreases with decreasing metallicity.

As a result of these effects, the X-ray optical depth is lower roughly by a factor of 2 . The use of lower X-ray optical depth in the calculation of Owocki \& Cohen (2006) leads to better agreement between predicted and observed X-ray line shapes. Conversely, the effect of scattering in these lines (Ignace \& Gayley 2002) might decrease with metallicity.

\subsection{Phosphorus line profiles}

The ion P V is assumed to be a dominant phosphorus ion in the stellar wind. Since its abundance is very low, its resonance lines as observed in the hot star wind spectra are generally unsaturated. Consequently, these lines are assumed not to be influenced by clumping and are ideal for determining the wind mass-loss rate. This was done by Fullerton et al. (2006), who showed that the mass-loss rate determined from the $\mathrm{PV}$ lines is much lower than when derived from the $\mathrm{H} \alpha$ line or radio emission.

This discrepancy can have two explanations: either P V is not a dominant phosphorus ion or the winds are clumped and their mass-loss rates are much lower. However, the phosphorus line profiles are sensitive to phosphorus abundance and to the overall stellar metallicity (due to the dependence of wind mass-loss rates on the metallicity). Both these effects lead to the weakening of $\mathrm{PV}$ line profiles. The phosphorus abundance changes by a factor of 0.81 when using a new abundance determination and, together with the decrease in wind mass-loss rate caused by the decrease in the metallicity, leads to the change in PV line optical depth by a factor of about 0.62 . Although this shift does not explain the main difference between theory and observations (possibly also the influence of clumping on the ionization balance has to be accounted for, Puls et al. 2006a), the use of new abundance determinations shifts the theoretical predictions closer to the observational results.

\section{Discussion and conclusions}

We have compared the wind models calculated using the older solar abundance determination (Anders \& Grevesse 1989) and the solar abundances derived using 3D hydrodynamical model atmospheres of Asplund et al. (2005). We have shown that the use of new abundances (which means lowering in the 
metallicity, but not uniformly for all elements) improves the agreement between observation and theory in several different aspects:

- Due to lower metallicity the predicted wind mass-loss rates are lower, consequently new models agree better with the mass-loss rates derived from observational analysis with clumping taken into an account.

- As a result of the lowering of mass-loss rates and slight lowering of terminal velocities, there is better agreement between the predicted modified wind momentum-luminosity relationship and that derived from observational analysis with clumping taken into account. On the other hand, if the clumping does not significantly influence the observed spectra, then the original good agreement between predicted wind momentum and the one derived from observation is worsened with models using improved solar abundance determination.

- Both the lower mass fraction of heavier elements and lower mass-loss rates lead to a decrease in the opacity in the X-ray region. This change is the most significant one because it basically combines the influence of two different effects (when using predicted mass-loss rates).

- Since both phosphorus abundance and the mass-loss rate are lower, there is better agreement between the predicted $\mathrm{PV}$ ionization fraction and the one derived from observations. However, lowering the wind density may partly compensate for this effect due to modification of the ionization structure.

We have to keep in mind that the actual metallicity of Galactic $\mathrm{O}$ stars may be different from the solar one. Moreover, it may be modified during the stellar evolution due to the rotational mixing (e.g., Maeder \& Meynet 2001), so our results have to be understood as a manifestation of the consequences of abundance changes in the theoretical wind models.

We can conclude that, although there is still not complete agreement between theory and observations, the use of more realistic solar abundance determinations may be a partial way to solve the problem because it improves the agreement in all aspects studied here.
Acknowledgements. This research made use of NASA's ADS and the SIMBAD database, operated at the CDS, Strasbourg, France. This work was supported by grant GA CR 205/04/1267. The Astronomical Institute Ondřejov is supported by the project AV0Z10030501.

\section{References}

Anders, E., \& Grevesse, N. 1989, Geochim. Cosmochim. Acta, 53, 197 Asplund, M. 2004, A\&A, 417, 769

Asplund, M., Grevesse, N., \& Sauval, A. J. 2005, Cosmic Abundances as Records of Stellar Evolution and Nucleosynthesis, ed. T. G. Barnes III, \& F. N. Bash (San Francisco: ASP), ASP Conf. Ser., 336, 25

Bouret, J.-C., Lanz, T., Hillier, D. J., et al. 2003, ApJ, 595, 1182

Castor, J. I. 1974, MNRAS, 169, 279

Chiosi, C., \& Maeder, A. 1986, ARA\&A, 24, 329

Fullerton, A. W., Massa, D. L., \& Prinja, R. K. 2006, ApJ, 637, 1025

Hummer, D. G., Berrington, K. A., Eissner, W., et al. 1993, A\&A, 279, 298

Ignace, R., \& Gayley, K. G. 2002, ApJ, 568, 954

Krtička, J. 2006, MNRAS, 367, 1282

Krtička, J., \& Kubát, J. 2004, A\&A, 417, 1003

Krtička, J., \& Kubát, J. 2006, Active OB-Stars: Laboratories for Stellar \& Circumstellar Physics, ed. S. Štefl, S. P. Owocki, \& A. T. Okazaki (San Francisco) ASP Conf. Ser., in press

Kudritzki, R. P., \& Puls, J. 2000, ARA\&A, 38, 613

Lamers, H. J. G. L. M., Snow, T. P., \& Lindholm D. M. 1995, ApJ, 455, 269 (LSL)

Maeder, A., \& Meynet, G. 2001, A\&A, 373, 555

Markova, N., Puls, J., Repolust, T., \& Markov, H., 2004, A\&A, 413, 693 (M04)

Martins, F., Schaerer, D., Hillier, D. J., et al. 2005, A\&A, 441, 735 (M05)

Nordlund, A., \& Dravins, D. 1990, A\&A, 228, 155

Oskinova, L. M., Feldmeier, A., \& Hamann, W.-R. 2006, MNRAS, 372, 313

Owocki, S. P., \& Cohen, D. H. 2006, ApJ, 648, 565

Puls, J., Kudritzki, R.-P., Herrero, A., et al. 1996, A\&A, 305, 171

Puls, J., Markova, N., \& Scuderi, S. 2006a, Mass loss from stars and the evolution of stellar clusters, ed. A. de Koter, L. Smith, \& R. Waters, in press [arXiv: astro-ph/0607290]

Puls, J., Markova, N., Scuderi, S., et al. 2006, A\&A, 454, 625

Repolust, T., Puls, J., \& Herrero, A. 2004, A\&A, 415, 349 (R04)

Schaller, G., Schaerer, D., Meynet, G., \& Maeder, A. 1992, A\&AS, 96, 269

Seaton, M. J., Zeippen, C. J., Tully, J. A., et al. 1992, Rev. Mex. Astron. Astrofis., 23, 19

Steffen, M., Ludwig, H.-G., \& Freytag, B. 1995, A\&A, 300, 473

Vink, J. S., de Koter, A., \& Lamers, H. J. G. L. M. 2001, A\&A, 369, 574 